\title{
Continuum Goldstone spectrum of two-color QCD at finite density with staggered quarks
}

\author{
Jonas Wilhelm, ${ }^{1,2}$ Lukas Holicki, ${ }^{1}$ Dominik Smith $\odot,{ }^{1}$ Björn Wellegehausen, ${ }^{1}$ and Lorenz von Smekal ${ }^{1}$ \\ ${ }^{1}$ Institut für Theoretische Physik, Justus-Liebig-Universität, Heinrich-Buff-Ring 16, \\ 35392 Giessen, Germany \\ ${ }^{2}$ Institut für Kernphysik, Johannes-Gutenberg-Universität, Johann-Joachim-Becher-Weg 45, \\ 55099, Mainz, Germany
}

(Received 15 October 2019; published 10 December 2019)

\begin{abstract}
We carry out lattice simulations of two-color QCD and spectroscopy at finite density with two flavors of rooted-staggered quarks and a diquark source term. As in a previous four-flavor study [J. B. Kogut, D. Toublan, and D. K. Sinclair, The PseudoGoldstone spectrum of two color QCD at finite density, Phys. Rev. D 68, 054507 (2003).], for small values of the inverse gauge coupling we observe a Goldstone spectrum which reflects the symmetry-breaking pattern of a Gaussian symplectic chiral random-matrix ensemble (GSE) with Dyson index $\beta_{D}=4$, which corresponds to any-color QCD with adjoint quarks in the continuum instead of $\mathrm{QC}_{2} \mathrm{D}$ with fundamental quarks. We show that this unphysical behavior occurs only inside of the bulk phase of $S U(2)$ gauge theory, where the density of $Z_{2}$ monopoles is high. Using an improved gauge action and a somewhat larger inverse coupling to suppress these monopoles, we demonstrate that the continuum Goldstone spectrum of two-color QCD, corresponding to a Gaussian orthogonal ensemble (GOE) with Dyson index $\beta_{D}=1$, is recovered also with rooted-staggered quarks once simulations are performed away from the bulk phase. We further demonstrate how this change of random-matrix ensemble is reflected in the distribution of eigenvalues of the Dirac operator. By computing the unfolded level spacings inside and outside of the bulk phase, we demonstrate that, starting with the lowlying eigenmodes which determine the infrared physics, the distribution of eigenmodes continuously changes from the GSE to the GOE one as monopoles are suppressed.
\end{abstract}

DOI: 10.1103/PhysRevD.100.114507

\section{INTRODUCTION}

The QCD phase diagram continues to be the subject of intense theoretical and experimental studies. The region of high baryon density at relatively low temperatures is of particular relevance for the inner cores of neutron stars, and at somewhat higher temperatures for neutron-star mergers. It is probed experimentally in the beam-energy scan at RHIC and the future heavy-ion programs at J-PARC, NICA and FAIR. In this regime one usually expects a chiral firstorder transition ending a critical point, but inhomogeneous phases or more exotic states of matter like a quarkyonic phase have been proposed to occur as well. At even higher densities, beyond the reach of current experiments and astrophysical observations, asymptotic freedom and the attractive perturbative interactions between quarks close to

Published by the American Physical Society under the terms of the Creative Commons Attribution 4.0 International license. Further distribution of this work must maintain attribution to the author(s) and the published article's title, journal citation, and DOI. Funded by SCOAP . the Fermi surface entail the formation of Cooper pairs and color superconductivity.

Unfortunately, QCD at high densities remains inaccessible to stochastic integration methods, since the fermion determinant becomes complex at finite chemical potential $\mu$. This leads to an insurmountably hard fermion-sign problem precisely where a finite baryon density starts to build up in the ground state. The problem does not arise on the other hand in certain QCD-like theories, e.g., two-color $\mathrm{QCD}\left(\mathrm{QC}_{2} \mathrm{D}\right)$ or $\mathrm{G}_{2}-\mathrm{QCD}[1,2]$, which show chiral symmetry breaking, confinement and asymptotic freedom as well. These theories can thus be approached with standard Monte Carlo techniques on the lattice and provide therefore interesting test beds to develop and test algorithms for QCD at finite density.

Beside this technical aspect, QCD-like theories are interesting in their own right. On the lattice, $\mathrm{QC}_{2} \mathrm{D}$ has been studied with staggered [3-12] and Wilson fermions [13-18]. In contrast to QCD, the color-singlet baryons are diquarks and hence bosonic in two-color QCD, for example, while fermionic baryons do not exist in its spectrum. The physics of the bosonic diquark baryons qualitatively resembles QCD at finite isospin density with pion condensation [19] and is 


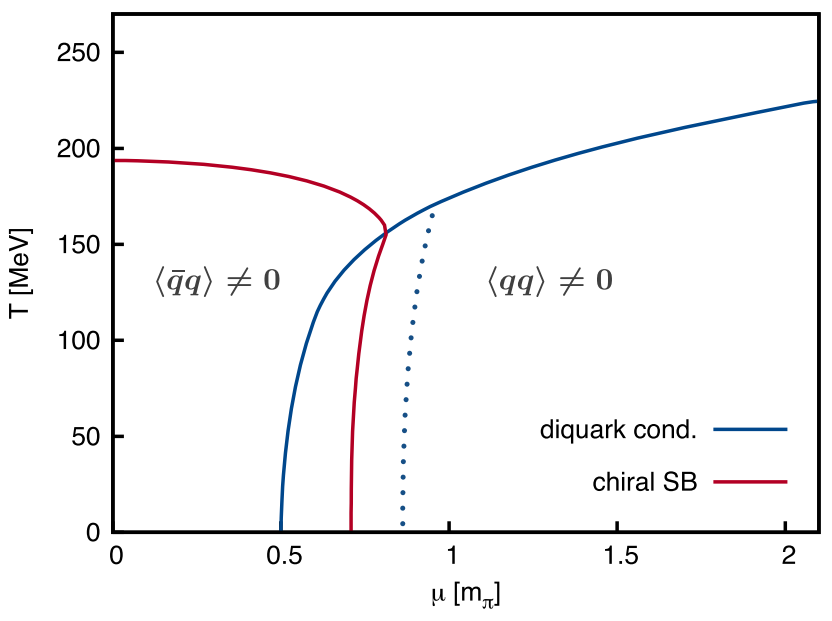

FIG. 1. Phase diagram of the quark-meson-diquark model for $\mathrm{QC}_{2} \mathrm{D}$ from the functional renormalization group [33,34]: halfvalue of the chiral condensate (red), and second-order phase boundary for diquark condensation (solid blue) with rough indication of the BEC-BCS crossover (dotted blue).

by now fairly well understood [20-23]. There are firm predictions for diquark condensation when $\mu$ reaches half the pion mass $m_{\pi}$ from chiral effective field theory and random matrix theory [6,24-30], and model studies of the BEC-BCS crossover inside the condensed phase [31-34]. In $\mathrm{QC}_{2} \mathrm{D}$ the lightest diquarks play a dual role as two-color baryons and pseudo-Goldstone bosons of the dynamical breaking of an extended chiral symmetry. When they condense, they are expected to form a superfluid which changes in nature from a Bose-Einstein condensate of tightly bound diquarks to a BCS-like pairing of quarks as chiral symmetry gets gradually restored with increasing density.

The phase diagram of the quark-meson-diquark model for $\mathrm{QC}_{2} \mathrm{D}$ from the functional renormalization group [33] is shown in Fig. 1. The qualitative features resemble lattice results $[16,17]$, especially when the Polyakov-loop variable is included in the effective model description [34]. Evidence of the BEC-BCS crossover inside the diquarkcondensation phase was also provided from lattice simulations [9] albeit still close to the bulk phase of $S U(2)$ (see below).

Antiunitary symmetries of the Dirac operators in QCDlike theories without a fermion-sign problem are both a blessing and a curse for the rooted-staggered fermion formulation. On one hand, phase ambiguities when rooting a complex determinant [35] do not occur. In fact, even for a single staggered fermion the determinant remains positive at finite $\mu$, whereas this requires two flavors of continuum Dirac fermions. This is because of the missing $C^{2}=-1$ from the charge conjugation matrix $C$ for Dirac spinors in the antiunitary symmetries of staggered Dirac operators. On the other hand, it implies that the corresponding Gaussian chiral random matrix ensembles get swapped, staggered fermions reflect the behavior of the Gaussian symplectic ensemble (GSE) when the continuum Dirac fermions show that of the Gaussian orthogonal ensemble (GOE) and vice versa. In particular, the staggered Dirac operator of fundamental quarks in $\mathrm{QC}_{2} \mathrm{D}$ has the GSE Dyson index $\beta_{D}=4$, while for continuum or Wilson fermions it is the GOE one, $\beta_{D}=1$. For adjoint quarks in any-color QCD (or fundamental quarks in $G_{2}-\mathrm{QCD}$ ) it is just the other way round. This is why the sector of positive fermion determinant of $\mathrm{QC}_{2} \mathrm{D}$ with adjoint quarks was studied as a replacement for the continuum theory within the correct random matrix ensemble in the early days [5].

Here we have addressed the following question: with the full $S U(4)$ taste symmetry in the continuum limit, it should be possible to define a standard (tasteless) charge conjugation from that for staggered quarks [36] as well. Does this imply that the correct symmetry breaking pattern, corresponding to the random matrix ensemble of the continuum two-color Dirac operator is recovered also with staggered quarks in the continuum limit?

The answer seems to be positive. The particular evidence for this that we provide is the behavior of the Goldstone pion inside the diquark condensation phase which shows the characteristic change indicative of the change of the Dyson index as the continuum limit is approached:

In the continuum, the extended $S U\left(2 N_{\mathrm{f}}\right)$ chiral symmetry is dynamically broken down to the compact symplectic $\operatorname{Sp}\left(N_{\mathrm{f}}\right)$ with fundamental quarks and Dyson index $\beta_{D}=1$ [25]. ${ }^{1}$ For $N_{\mathrm{f}}=2$ on the bosonic level this amounts to the simple vectorlike breaking of $S O(6) \rightarrow$ $S O(5)$ with coset $S^{5}$ and five Goldstone bosons, three pions and a scalar (anti)diquark pair. The exact chiral symmetry extending the usual $U(1)_{e} \times U(1)_{o}$ of the staggered action in the two-color case is $U(2)$ on the other hand [4]. With fundamental quarks it breaks down to $U(1)_{V}$. Up to the Goldstone pion of the broken $U(1)_{\epsilon}$ it therefore resembles the $N_{\mathrm{f}}=1$ case of continuum quarks with Dyson index $\beta_{D}=4$ as in adjoint QCD [25] or $G_{2}$-QCD [37] which is $S U(2) \rightarrow U(1){ }^{2}$

The effective field theory prediction for the Goldstone spectrum with the $U(2) \rightarrow U(1)$ chiral symmetry breaking of the fundamental staggered two-color action was explicitly worked out in [3]. Most important for our purposes is the behavior of the Goldstone pion inside the diquark condensation phase at $\mu \geq m_{\pi} / 2$ where it resembles that of the symmetric pion branch $P_{S}$ of the Dyson index $\beta_{D}=4$ case in the continuum [25], with a mass that decreases $\sim m_{\pi}^{2} / 2 \mu$, although this branch strictly speaking only exists for $N_{\mathrm{f}} \geq 2$ there.

\footnotetext{
${ }^{1}$ For adjoint quarks in any-color QCD or fundamental quarks in $G_{2}$-QCD with Dyson index $\beta_{D}=4$ in the continuum it breaks down to $\operatorname{spin}\left(2 N_{\mathrm{f}}\right)$, the double cover of $S O\left(2 N_{\mathrm{f}}\right)$.

${ }^{2}$ For adjoint staggered quarks in $\mathrm{QC}_{2} \mathrm{D}$ one has $U(2) \rightarrow$ $S p(1) \sim S U(2)$ and hence only the Goldsone pion of the broken $U(1)_{\epsilon}$ corresponding to having no Goldstone bosons with Dyson index $\beta_{D}=1$ for $N_{\mathrm{f}}=1$ in the continuum theory.
} 
We will demonstrate that the behavior of this Goldstone pion branch in the continuum limit indeed changes to that of the symmetric $P_{S}$ mode in the $\beta_{D}=1$ case of the continuum theory which increases $\sim 2 \mu$ inside the diquark condensation phase [25]. Again this mode exists in the continuum only for $N_{\mathrm{f}} \geq 2$, starting with a multiplicity of 3 for the three degenerate pions in the two-flavor case. This again indicates that the taste symmetry needs to be at least partially restored to achieve this.

Here it is also important to note that the previous results with staggered quarks in $\mathrm{QC}_{2} \mathrm{D}$ that led to the $\beta_{D}=4$ Goldstone spectrum were obtained inside a so-called bulk phase on the lattice, where the lattice spacing is almost independent of the inverse gauge coupling. The large effects of this on spectroscopy and thermodynamics at vanishing chemical potential have been investigated in Ref. [38]. The influence of bulk effects on simulations at finite density has not yet been discussed and is thus the main focus of our study. Indeed, we will find that inside the bulk phase, the Goldstone spectrum for Dyson index $\beta_{D}=$ 4 as for adjoint continuum quarks is reproduced, while outside the bulk phase we observe the correct Goldstone spectrum of two-color QCD, corresponding to the Dyson index $\beta_{D}=1$ of the continuum Dirac operator, also with rooted-staggered quarks. We demonstrate that this change of Dyson index is reflected in the eigenvalue statistics of the Dirac operator. By computing the unfolded level spacings, we demonstrate that the distribution of eigenmodes is completely dominated by the GSE in the bulk phase but obtains larger contributions from the GOE as one leaves the bulk phase. This change turns out to be continuous and builds up starting with the low-lying eigenmodes. As infrared physics is controlled by these lower levels, we observe that one correctly reproduces the continuum theory Goldstone spectrum even when the higher eigenmodes are still dominated by the GSE.

This paper is organized as follows: In Sec. II we introduce the lattice action, including a diquark source term, and the simulation parameters used in this work. In Sec. III we study the quark-number density and chiral and diquark condensates for small values of the gauge coupling (which turns out to be inside the bulk phase) and compare our results to the predictions of leading order chiral perturbation theory. Section IV explains the bulk phase and introduces its order parameter, the $Z_{2}$-monopole density, ending with a discussion on new lattice parameters to suppress bulk effects. In Sec. V we then repeat the study of Sec. III with our new set of parameters and discuss lattice discretization and finite volume effects, such as the effective quenching of the theory in the saturated regime and problems with additive renormalization of the chiral condensate at finite $\mu$. In Sec. VI after providing a detailed description of chiral symmetry-breaking pattern of staggered fermions, we present our main result, which is the numerical measurement and comparison of the Goldstone spectrum at nonvanishing chemical potential, inside and outside the bulk phase. And finally, in Sec. VII we present the unfolded level spacings of the Dirac operator and demonstrate that change of Goldstone spectrum is accompanied by a change of eigenvalue distribution. We end with our conclusion and an outlook in Sec. VIII.

\section{LATTICE SETUP}

For the low-temperature scan of the Goldstone spectrum of two-color QCD, we use standard rooted staggered fermions, with the Dirac operator,

$$
\begin{aligned}
D_{x, y}(\mu)= & a m \delta_{x, y}+\sum_{\nu=1}^{4} \frac{\eta_{\nu}(x)}{2 a}\left(e^{a \mu \delta_{\nu, 4}} U_{\nu}(x) \delta_{x+\hat{\nu}, y}\right. \\
& \left.-e^{-a \mu \delta_{\nu, 4}} U_{\nu}^{\dagger}(x-\hat{\nu}) \delta_{x-\hat{\nu}, y}\right),
\end{aligned}
$$

at nonvanishing baryon chemical potential $\mu$. To study spontaneous symmetry breaking and competing order on a finite lattice, we add a diquark source $\lambda$ corresponding to a Majorana mass term in the Lagrangian, which explicitly breaks the chiral $U(2)$ symmetry of the massless staggered action down to a $U(1)$ as well but in a direction different from that of the Dirac mass term [4-7],

$$
S_{\mathrm{f}}=\bar{\chi} D(\mu) \chi+\frac{\lambda}{2}\left(\chi^{T} \tau_{2} \chi+\bar{\chi} \tau_{2} \bar{\chi}^{T}\right) .
$$

Physical results are then retrieved in the $\lambda \rightarrow 0$ limit. The diquark condensate is obtained from

$$
\langle q q\rangle=\left.\left\langle\chi^{T} \tau_{2} \chi\right\rangle \propto \frac{\partial \ln Z}{\partial \lambda}\right|_{\lambda \rightarrow 0} .
$$

The staggered fermion action is conveniently expressed in a Nambu-Gorkov basis,

$$
S_{\mathrm{f}}=\frac{1}{2}\left(\bar{\chi}, \chi^{T} \tau_{2}\right) A\left(\begin{array}{c}
\tau_{2} \bar{\chi}^{T} \\
\chi
\end{array}\right),
$$

with an inverse Nambu-Gorkov propagator,

$$
A=\left(\begin{array}{cc}
\lambda & D(\mu) \\
-D^{\dagger}(\mu) & \lambda
\end{array}\right) .
$$

Grassmann integration produces the square root of the determinant of $A$ in the path integral measure. This is seen most easily when considering $\left(\chi, \bar{\chi}^{T}\right)$ as a set of independent real Grassmann variables, whose Gaussian integral results in a (positive) Pfaffian which agrees with $\sqrt{\operatorname{det} A}$, where

$$
\operatorname{det} A=\operatorname{det}\left(D^{\dagger}(\mu) D(\mu)+\lambda^{2}\right) .
$$

In fact, a direct use of hybrid Monte Carlo (HMC) based on a Gaussian pseudofermion integral over $\left(A A^{\dagger}\right)^{-1}$ would even produce $(\operatorname{det} A)^{2}$ in the measure. However, because 


$$
A^{\dagger} A=A A^{\dagger}=\left(\begin{array}{cc}
D(\mu) D(\mu)^{\dagger}+\lambda^{2} & 0 \\
0 & D^{\dagger}(\mu) D(\mu)+\lambda^{2}
\end{array}\right)
$$

is block diagonal with both blocks having the same determinant, we can use size-half pseudofermion fields (in the Nambu-Gorkov space) to remove this further doubling. On the other hand, we can not use size-half fields in the even-odd staggered lattice at finite $\mu$. This means that without any rooting, we compute $\operatorname{det} A$ and describe eight fermion species instead of the usual four staggered tastes. Therefore we use standard rooting techniques to compute $(\operatorname{det} A)^{N_{\mathrm{f}} / 8}$ for each continuum flavor; i.e., we take the fourth root to simulate with $N_{\mathrm{f}}=2$ as in Ref. [9]. The rooting is achieved by a rational approximation of the fermion matrix in the pseudofermion action of the HMC algorithm.

The diquark source explicitly breaks baryon number conservation, or more precisely the $U(1)_{V}$ of the staggered action, and hence in addition to the usual $\langle\psi \gamma \bar{\psi}\rangle$ contractions in the calculation of the correlation functions, we now also have $\langle\psi \gamma \psi\rangle$ and $\langle\bar{\psi} \bar{\psi}\rangle$ contractions. These correspond to the diagonal terms of the propagator $G=A^{-1}$ obtained from Eq. (5),

$$
G=\left(\begin{array}{cc}
\left(D D^{\dagger}+\lambda^{2}\right)^{-1} \lambda & -\left(D D^{\dagger}+\lambda^{2}\right)^{-1} D \\
\left(D^{\dagger} D+\lambda^{2}\right)^{-1} D^{\dagger} & \left(D^{\dagger} D+\lambda^{2}\right)^{-1} \lambda
\end{array}\right) .
$$

In this paper we compare results with $N_{\mathrm{f}}=2$ staggered flavors at $\beta=1.5$ on a $12^{3} \times 24$ lattice with am $=0.025$, which turns out to be deep inside the bulk phase, to $N_{\mathrm{f}}=2$ staggered flavors at $\beta=1.7$ on a $16^{3} \times 32$ lattice with $a m=0.01$ and an improved gauge action so that this is just outside the bulk phase. The parameters of the simulations at $\beta=1.5$ with the unimproved gauge action correspond to those used in Ref. [3] for $N_{\mathrm{f}}=4$.

\section{EFFECTIVE FIELD THEORY PREDICTIONS}

Kogut et al. have studied the symmetries of QCD-like theories at finite baryon density with pseudoreal quarks in the fundamental representation using chiral effective Lagrangians $[3,25]$. A linear sigma model for the symmetry-breaking pattern and Goldstone spectrum of the staggered two-color action was used to describe the data in [3]. For the purpose of illustrating the basic features of the diquark-condensation transition at $\mu=\mu_{c}=m_{\pi} / 2$ here we fit our data to the somewhat simpler form of the leadingorder chiral perturbation theory $(\chi \mathrm{PT})$ predictions from the nonlinear sigma model [25]. This describes the rotation of the vacuum alignment from the chiral $\langle\bar{q} q\rangle$ into the diquark $\langle q q\rangle$ condensate at a fixed

$$
\Sigma_{\mathrm{c}}=\sqrt{\langle\bar{q} q\rangle^{2}+\langle q q\rangle^{2}} \equiv 2 N_{\mathrm{f}} G .
$$



FIG. 2. Fit of lattice data to leading-order $\chi \mathrm{PT}$ form of chiral (purple) and diquark (orange) condensates, and quark-number density (brown) from Eqs. (9) and (10), with $N_{\mathrm{f}}=2, \beta=1.5$, $a m=0.025, a \lambda=0.0025$ on a $12^{3} \times 24$ lattice with unimproved Wilson gauge action.

With explicit diquark source $\lambda$, the rotation angle $\alpha(\mu)$ is obtained from

$$
\mu^{2} \cos \alpha \sin \alpha=\mu_{c}^{2}\left(\sin \alpha-\frac{\lambda}{m} \cos \alpha\right),
$$

such that a nonzero value $\alpha_{0}$ is obtained already at $\mu=0$ which depends on the relative size of the Majorana and Dirac quark masses, i.e., $\tan \alpha_{0}=\lambda / m$. Chiral and diquark condensate, and quark-number density $n$ as functions of $\mu$ are then given by

$$
\begin{aligned}
\langle\bar{q} q\rangle & =2 N_{\mathrm{f}} G \cos \alpha, \\
\langle q q\rangle & =2 N_{\mathrm{f}} G \sin \alpha, \\
n & =8 N_{\mathrm{f}} F^{2} \mu \sin ^{2} \alpha .
\end{aligned}
$$

As a first test we have performed simulations with the parameters of Ref. [3], i.e., a lattice gauge coupling of $\beta=1.5$, quark mass $a m=0.025$, and diquark source $a \lambda=$ 0.0025 on a $12^{3} \times 24$ lattice and the standard Wilson plaquette action, so as to reproduce their results with the square root of the determinant in (6) for $N_{\mathrm{f}}=4$.

With the fourth root for $N_{\mathrm{f}}=2$ continuum flavors and the same lattice parameters we have then obtained the results shown in Fig. 2. They are fitted to the leading-order forms from chiral perturbation theory in Eq. (10) with (9) for the vacuum alignment angle $\alpha(\mu)$. From these fits we obtain the fit parameters $F, G$, and the critical chemical potential, which results as $a \mu_{\mathrm{c}}=0.1889(5)$. Within the errors this agrees with our spectroscopic result from the pion correlator at $\lambda=0.0025, \mu=0$ which yields $a m_{\pi} / 2=0.1887(6)$. The agreement between fits and data in Fig. 2 is nearly perfect up to $a \mu \sim 0.3$.

The deviations at larger chemical potentials were attributed to a $\mu$-dependence of the total condensate $\Sigma_{\mathrm{c}}$ in 
Ref. [3] where linear sigma model fits were therefore used instead. These imply $\langle q q\rangle \sim \mu$ and hence $n \sim \mu^{3}$ at large $\mu$, as predicted for a BCS-like pairing in QCD at large isospin density already in [19]. The corresponding rise in the isospin density beyond the $\chi \mathrm{PT}$ prediction was observed in lattice QCD simulations [20], and it was traced to the BEC$\mathrm{BCS}$ crossover in a functional renormalization group study of the quark-meson model as an effective theory with linearly realized chiral symmetry and order-parameter fluctuations beyond mean field [21].

The same interpretation of this rise in the diquark density, beyond the $\chi \mathrm{PT}$ prediction, as an indication of the BEC-BCS crossover in $\mathrm{QC}_{2} \mathrm{D}$, was also adopted in Ref. [9]. Because these results were obtained on rather coarse lattices, it is therefore important to verify that they are not qualitatively affected by strong discretization artifacts such as the $Z_{2}$ monopoles in the bulk phase of $S U(2)$.

\section{THE BULK TRANSITION}

Most lattice gauge theories, as for instance $S U(2)$, $S U(3)$ or $G_{2}$ gauge theory, exhibit a bulk phase in the strong-coupling regime, characterized by the presence of unphysical lattice artifacts such as electric vortices and magnetic monopoles [39-44]. These dominate the ultraviolet behavior, such that the lattice spacing is nearly independent of the coupling constant, and taking a continuum limit is not possible. In the physical weak-coupling regime the short distance physics is governed by asymptotic freedom and the continuum limit is approached by $\beta \rightarrow \infty$. Depending on which gauge group and which representation, both regions are either separated by a true phase boundary or a crossover, the later being the case for the fundamental representation of $S U(2)$ considered here (cf. Fig. 1 in Ref. [43]). The bulk transition is almost independent of the lattice size and persists also in the presence of fermions $[45,46]$.

An order parameter for the strong-coupling to weakcoupling transition is the $Z_{2}$ monopole density [41],

$$
\langle z\rangle=1-\frac{1}{N_{C}} \sum_{C} \prod_{P \in \partial C} \operatorname{sgn} \operatorname{tr} P,
$$

where $\sum_{C}$ runs over all elementary cubes of the lattice. $\langle z\rangle$ is sensitive to preferred signs of the plaquettes on the faces of these cubes, which are aligned below the bulk transition: In the bulk phase $\langle z\rangle$ is nonvanishing, while it vanishes in the physical weak-coupling regime.

With $\beta=1.5$ and the unimproved Wilson gauge action, we have found the $Z_{2}$ monopole density at $a \mu=0$ to be $\langle z\rangle=0.884040(95)$, and thus we expect bulk effects to be dominant in this regime. We have also confirmed that $\langle z\rangle$ is only very weakly affected by the inclusion of dynamical quarks. For a fixed inverse gauge coupling $\beta$, the monopole density can be significantly reduced with Symanzik's gauge action [47] (we employ the tree-level improved

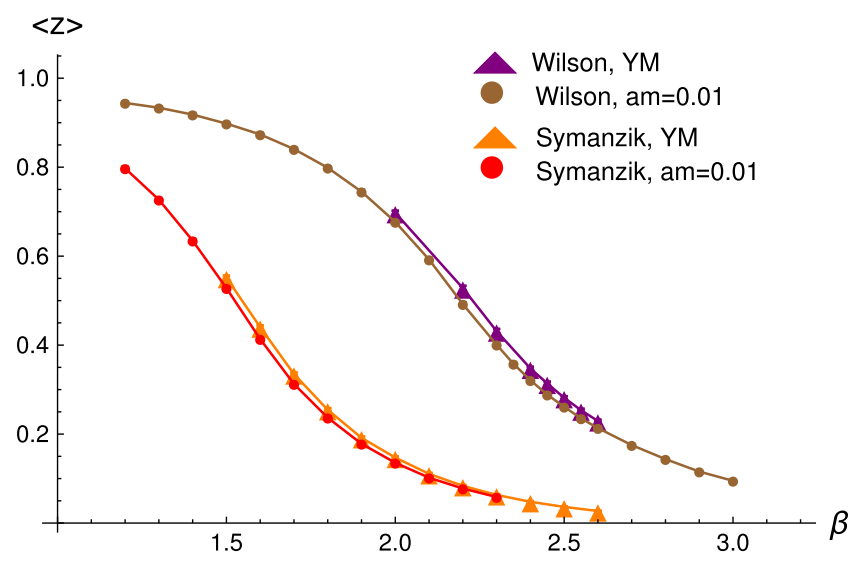

FIG. 3. Comparison of the $Z_{2}$ monopole density on a $N_{s}=12$, $N_{t}=8$ lattice for Wilson and tree-level improved Symanzik gauge action in pure Yang-Mills theory and with standard staggered fermions for $a m=0.01$ as a function of the inverse gauge coupling $\beta$.

variant here). This is illustrated in Fig. 3, which shows the $\beta$-dependence of $\langle z\rangle$ for the improved and unimproved actions, with and without dynamical fermions in each case.

In principle, one would like to suppress $Z_{2}$ monopoles as much as possible. In practice, finite volume effects become increasingly severe at larger $\beta$ due to a smaller physical lattice spacing $a$, and one is forced to make a compromise. This is illustrated in Fig. 4, which shows the $\beta$-dependence of various meson masses obtained in a previous study [38]. For small physical volumes $(a N)^{4}$ (larger $\beta$ ) the meson masses degenerate, signaling an explicit breaking of chiralsymmetry by the finite system size, while for small inverse couplings $\beta$ bulk effects are dominant. For our simulations of the continuum physics we therefore chose $\beta=1.7$ with $N_{s}=16$ and $N_{t}=32$, as a compromise between small $a$

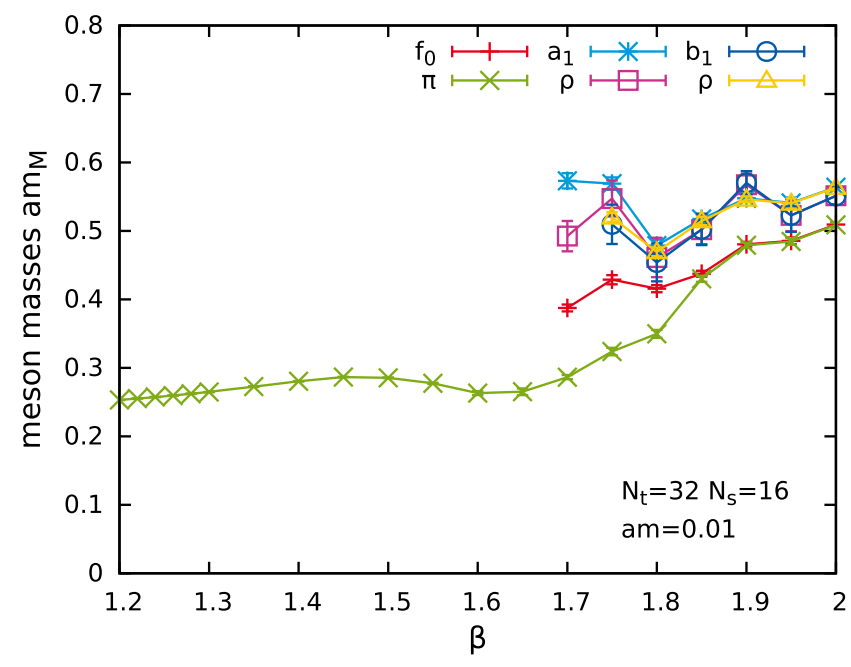

FIG. 4. $\quad \beta$-dependence of meson masses, obtained with standard staggered fermions and tree-level improved Symanzik gauge action. Figure taken from [38]. 
and small $\beta$, where $m_{\pi} / m_{\rho}=0.58(5)$. Using the improved action the $Z_{2}$ monopole density at $\beta=1.7$ and $a \mu=0$ is $\langle z\rangle=0.27340(66)$. Although there is still a substantial amount of monopoles on the lattice, this choice of parameters pushes the simulations on the weak-coupling side of the bulk crossover, as our spectroscopic results discussed below clearly demonstrate.

\section{LEAVING THE BULK PHASE}

Simulating at finite $\mu$ with the improved action and our new choice of lattice parameters $(\beta=1.7, a m=0.01$, $16^{3} \times 32$ ), we carry out a study of the $\mu$ dependence of different observables outside of the bulk phase. We first observe that we can again fit the quark-number density and diquark condensate to leading-order $\chi \mathrm{PT}$ predictions from Eqs. (9) and (10) (note here that the same expressions are predicted by $\chi \mathrm{PT}$ for both the Gaussian orthogonal and Gaussian symplectic ensembles [3,25]). We simulate with three different values of the explicit diquark source $a \lambda=0.0050 ; 0.0025 ; 0.0010$ and apply the fits directly at finite $\lambda$. Results are shown in Fig. 5, together with extrapolations to $\lambda \rightarrow 0$.

We find that our results for $a \lambda=0.0010$ already agree within one standard deviation with the limit of vanishing diquark source. Attempting to extract the critical chemical potential for diquark condensation from fits to $a \lambda=0.0010$ yields $a \mu_{\mathrm{c}}=0.172(21)$ however, which is slightly overestimated compared to our spectroscopic result $\left[a \mu_{\mathrm{c}}=\right.$ $0.1456(28)]$ from the pion correlator at $a \lambda=0.0010$, $\mu=0$. We find that a consistent value $\left[a \mu_{\mathrm{c}}=0.1356(86)\right]$ is obtained from a $\chi \mathrm{PT}$ fit to the $\lambda \rightarrow 0$ extrapolation of $\langle q q\rangle$. $^{3}$ We also observe significant deviations from the $\chi \mathrm{PT}$ predictions at around $a \mu \sim 0.3$, which we interpret as signaling the onset of the BEC-BCS crossover. We thus conclude that the behavior of $\langle q q\rangle$ and $\langle n\rangle$ is not qualitatively different outside of the bulk phase.

On the other hand, from an observed decrease of the chiral condensate above $\mu_{\mathrm{c}}$ (shown in Fig. 6), which we did not observe at $\beta=1.5$, we infer the presence of UV-divergence, such that renormalization is required. As discussed in Ref. [48], it is possible to renormalize the chiral condensate at finite temperature using the chiral susceptibility $\chi_{m_{q}}$, as both contain the same UV-divergent term $c_{\mathrm{UV}}$, viz.

$$
\begin{aligned}
\langle\bar{q} q\rangle_{m_{q}} & =\langle\bar{q} q\rangle_{0}+c_{2} m_{q}+\frac{c_{\mathrm{UV}}}{a^{2}} m_{q}+\mathcal{O}\left(m_{q}^{2}\right), \\
\chi_{m_{q}} & =c_{2}+\frac{c_{\mathrm{UV}}}{a^{2}}+\mathcal{O}\left(m_{q}^{2}\right) .
\end{aligned}
$$

\footnotetext{
${ }^{3}$ In Ref. [23] it was shown how $\lambda \rightarrow 0$ extrapolations can be improved using singular values of the Dirac operator. We did not pursue this however, as a simple fitting procedure turns out to be sufficient for our purpose.
}

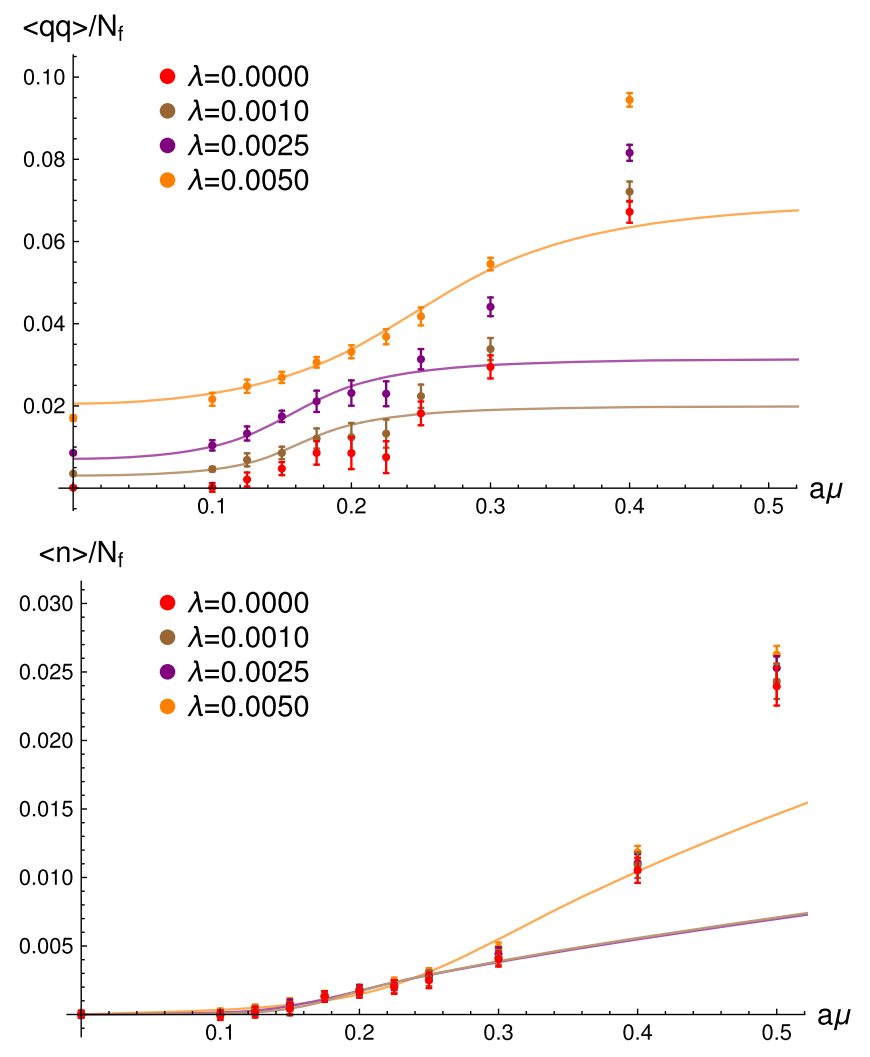

FIG. 5. Fit of lattice data to leading-order $\chi \mathrm{PT}$ form of diquark condensate (top) and quark-number density (bottom) from Eqs. (9) and (10), with $N_{\mathrm{f}}=2, \beta=1.7, a m=0.01, a \lambda=$ $0.0050 ; 0.0025 ; 0.0010$ on a $16^{3} \times 32$ lattice with tree-level improved Symanzik gauge action. Points for $a \lambda=0.0$ obtained by extrapolation.

Since the UV divergence originates mainly from the connected chiral susceptibility $\chi^{\text {con }}$ (also shown in Fig. 6, we neglect $\lambda$-dependent contributions here), a renormalized condensate can be defined as $\Sigma=\langle\bar{q} q\rangle_{m_{q}}-m_{q} \chi^{\text {con. }}$. We observe that both the condensate and $\chi^{\text {con }}$ exhibit a similar decrease at large $\mu$ and thus conclude that the UV-divergence $c_{\mathrm{UV}}$ is $\mu$-dependent.

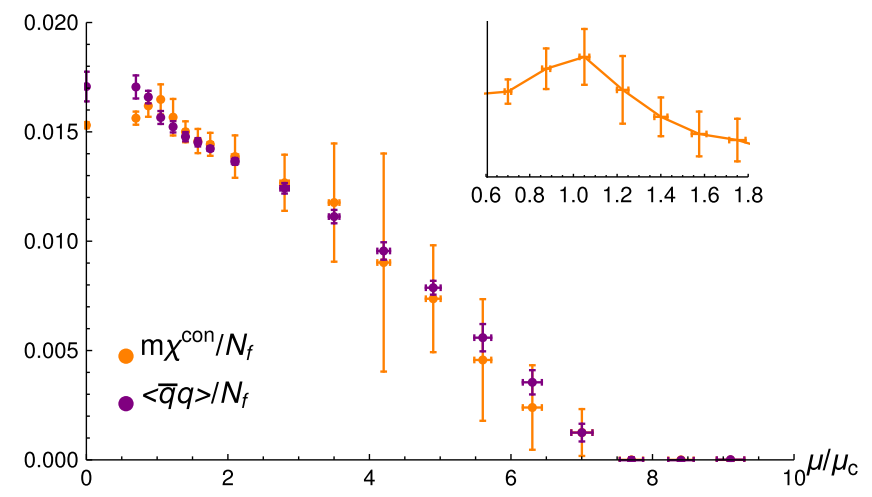

FIG. 6. Chiral condensate and connected susceptibility, $16^{3} \times 32, a m=0.01, \beta=1.7, a \lambda=0.005$ with a zoom to the singular contribution to $\chi^{\text {con }}$. 




FIG. 7. Volume dependence of the connected chiral susceptibility for $16^{3} \times 32$, am $=0.01, \beta=1.7, a \lambda=0.005$.

At the chiral transition the disconnected susceptibility $\chi^{\text {dis }}$ contains a singular contribution. At the diquark condensation transition, we find that $\chi^{\text {con }}$ has a singular part as well, as its peak height is bounded from above by a finite volume (see Fig. 7). This singularity will dominate over $c_{\mathrm{UV}} / a$ at finite $a$ in the infinite volume limit. The chiral condensate on the other hand, at zero temperature, must remain independent of $\mu$ for $\mu<\mu_{c}$. It does not have such a singular contribution, and it would be unphysical to introduce one with the connected susceptibility subtraction. At any rate, this would introduce a $\mu$-dependence below $\mu_{c}$ and hence a Silver-Blaze problem. Therefore, a different ( $\mu$-dependent) subtraction of the chiral condensate is required. Likewise, it is impossible to remove the UV-divergence by subtracting a heavy quark condensate like $\langle\bar{q} q\rangle_{m_{q}}-\frac{m_{q}}{m_{q}^{\prime}}\langle\bar{q} q\rangle_{m_{q}^{\prime}}$, since the pion mass and thus the position of the diquark onset strongly depend on the quark mass.

Measuring the $\mu$ dependence of the $Z_{2}$ monopole density and the quark number density, we observe that both quantities saturate at large $\mu$ (see Figs. 8 and 9). In these figures, $\mu$ has been normalized with the critical chemical potential $\mu_{\mathrm{c}}=m_{\pi} / 2$. With increasing $\mu$ the $Z_{2}$ monopole density approaches its quenched value, while at the same

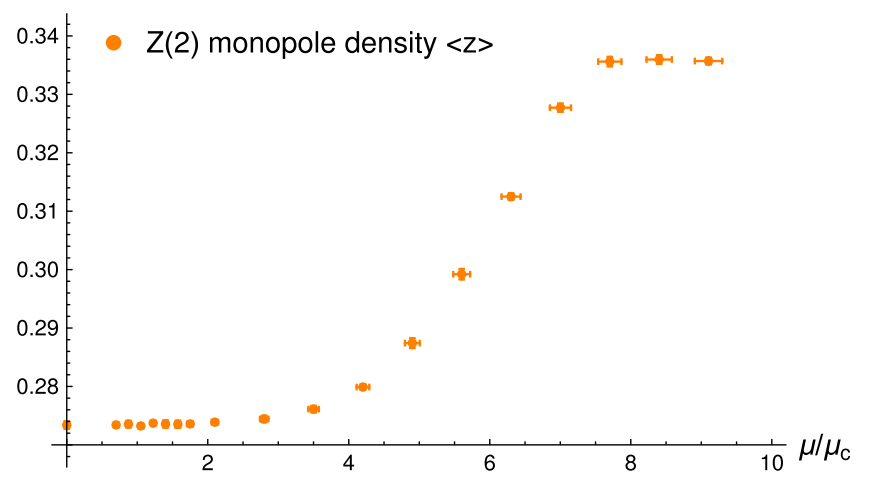

FIG. 8. $Z_{2}$ monopole density for staggered quarks with $|\Lambda|=16^{3} \times 32, a m=0.01, a \lambda=0.001$ and $\beta=1.7$.

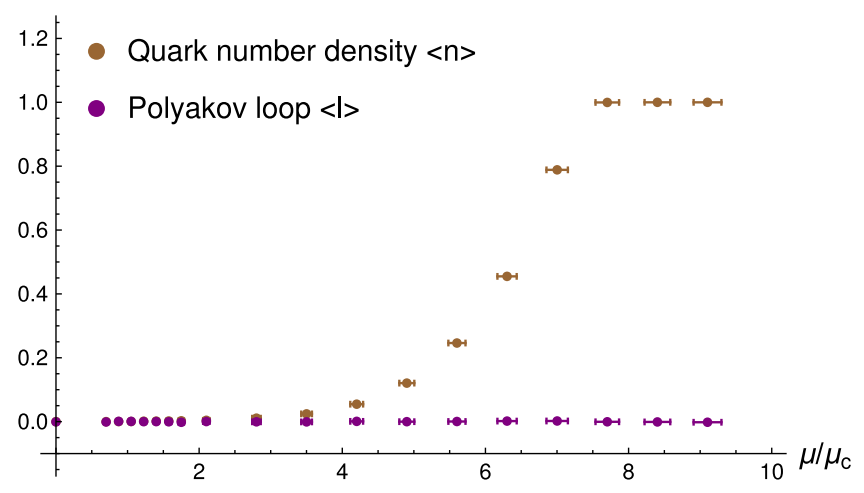

FIG. 9. Quark number density and Polyakov loop for staggered quarks with $|\Lambda|=16^{3} \times 32$, am $=0.01, a \lambda=0.001$ and $\beta=1.7$.

point the quark number density saturates. We conclude that in this high chemical potential regime the lattice is fully occupied with fermions, such that the system effectively becomes quenched.

Finally, we observe that the Polyakov loop is rather insensitive to the chemical potential with staggered quarks and in fact coincides with its value in the quenched limit (see Fig. 9). This is in contrast to lattice simulations of two-color QCD with Wilson fermion $[10,16]$ and $G_{2}$-QCD with Wilson fermions [1], where the Polyakov loop shows a peak around half filling. Also, in a previous effective Polyakov loop model study for QCD-like theories [49,50] with heavy Wilson quarks it has been seen that the Polyakov loop expectation value has a peak at the inflection point of the quark number density.

To explain this discrepancy, we consider that in twodimensional two-color QCD, where large temporal extends of the lattice are feasible, the peak vanishes in the limit of $N_{t} \rightarrow \infty$ [51], suggesting that the nonvanishing Polyakov loop might be an effect of the residual temperature due to the finite lattice volume. This is also in agreement with recent lattice simulations with staggered fermions at zero temperature but larger inverse gauge coupling, leading to a larger residual temperature [52]. Here the Polyakov loop also increases with increasing chemical potential. For Wilson fermions, a larger lattice spacing might lead to the excitement of heavy doublers beyond some $a \mu$, such that the free energy becomes finite. As these heavy doublers are not present in the staggered formalism, this behavior is not observed here at comparable lattice spacings.

\section{CHIRAL SYMMETRY BREAKING PATTERN AND THE GOLDSTONE SPECTRUM}

Having established a set of parameters $(\beta=1.7$, $\left.16^{3} \times 32, a m=0.01\right)$ with which we expect to reproduce the continuum physics, we now turn to the primary focus of this work which is to study the Goldstone spectrum. We begin by reviewing the symmetry-breaking channels of the staggered action of two-color lattice QCD and discussing 
the associated Goldstone modes and correlation functions, which will then be compared inside and outside of the bulk phase. These issues were previously discussed in Ref. [4]. We present a compact summary here to keep this paper self-contained.

For this purpose, it is convenient to introduce a new basis for the fermion fields, given by

$$
\bar{X}_{e}=\left(\begin{array}{ll}
\bar{\chi}_{e} & -\chi_{e}^{T} \tau_{2}
\end{array}\right), \quad X_{o}=\left(\begin{array}{c}
\chi_{o} \\
-\tau_{2} \bar{\chi}_{o}^{T}
\end{array}\right),
$$

which separates even and odd sites. The kinetic part of the staggered action (4) then reads [4]

$$
\begin{aligned}
S_{\mathrm{kin}}= & \sum_{n \in \Lambda^{\prime}, \nu} \frac{\eta_{\nu}(n)}{2}\left[\bar{X}_{e}(n)\left(\begin{array}{cc}
e^{\mu \delta_{\nu, 4}} & 0 \\
0 & e^{-\mu \delta_{\nu, 4}}
\end{array}\right) U_{\nu}(n) X_{o}(n+\hat{\nu})\right. \\
& \left.-\bar{X}_{e}(n)\left(\begin{array}{cc}
e^{-\mu \delta_{\nu, 4}} & 0 \\
0 & e^{\mu \delta_{\nu, 4}}
\end{array}\right) U_{\nu}(n-\hat{\nu})^{\dagger} X_{o}(n-\hat{\nu})\right]
\end{aligned}
$$

where the sum runs over even sites only. It follows that in the limit $m=\lambda=\mu=0$ the fermion action is invariant under

$$
X_{o} \rightarrow V X_{o}, \quad \bar{X}_{e} \rightarrow \bar{X}_{e} V^{\dagger} ; \quad V \in U(2) .
$$

The original $U(1)_{e} \times U(1)_{o}$ symmetry of the $N_{f}=1$ staggered action for two-color QCD is therefore enlarged to $U(2)$ in this limit $[3,4,7]$.

Applying the same basis transformation to the mass and the diquark source terms one obtains

$$
\begin{aligned}
& \bar{\chi} \chi=\frac{1}{2}\left[\bar{X}_{e} \sigma_{1} \tau_{2} \bar{X}_{e}^{T}+X_{o}^{T} \sigma_{1} \tau_{2} X_{o}\right], \\
& \chi \chi=\frac{1}{2}\left[\bar{X}_{e} \sigma_{3} \tau_{2} \bar{X}_{e}^{T}+X_{o}^{T} \sigma_{3} \tau_{2} X_{o}\right],
\end{aligned}
$$

where we understand Pauli matrices $\sigma_{i}$ to act in the basis (13) and $\tau_{i}$ to act on color indices. Hence, the condensates are indistinguishable at $\mu=0$ as they are connected by $V=$ $\frac{i}{\sqrt{2}}\left(\mathbf{1}+i \sigma_{2}\right) \in U(2)$ [4]. The Goldstone modes are derived by applying infinitesimal $U(2)$ rotations,

$$
V_{\delta}=\mathbf{1}+i \delta \lambda, \quad \lambda \in\left\{\mathbf{1}, \tau_{i}\right\}
$$

to $\bar{\chi} \chi$ and $\chi \chi$, where the coefficient of $O(\delta)$ is then identified as the Goldstone mode [4]. The results are shown in Table I.

Both condensates leave one generator of $U(2)$ unbroken and hence induce the same symmetry-breaking pattern $U(2) \rightarrow U(1)$ at $\mu=0$. Since at $\mu \neq 0$ the symmetry is reduced from $U(2)$ to $U(1)_{e} \times U(1)_{o}$, one is left with two generators $\left\{\mathbf{1}, \sigma_{3}\right\}$ of $U(2)$, which correspond to the
TABLE I. The Goldstone modes for $N_{\mathrm{f}}=1$ according to the generators of $U(2)$, where $\epsilon$ corresponds to $\epsilon(n)=\eta_{5}(n)=$ $(-1)^{n_{1}+n_{2}+n_{3}+n_{4}}$.

\begin{tabular}{lcc}
\hline \hline & $\langle\bar{q} q\rangle$ & $\langle q q\rangle$ \\
\hline $\mathbf{1}$ & $\bar{\chi} \epsilon \chi$ & $\chi^{T} \tau_{2} \epsilon \chi+\bar{\chi} \tau_{2} \epsilon \bar{\chi}^{T}$ \\
$\tau_{1}$ & $\chi^{T} \tau_{2} \chi-\bar{\chi} \tau_{2} \bar{\chi}^{T}$ & $\cdots$ \\
$\tau_{2}$ & $\chi^{T} \tau_{2} \chi+\bar{\chi} \tau_{2} \bar{\chi}^{T}$ & $\bar{\chi} \chi$ \\
$\tau_{3}$ & $\cdots$ & $\chi^{T} \tau_{2} \chi-\bar{\chi} \tau_{2} \bar{\chi}^{T}$ \\
\hline \hline
\end{tabular}

staggered $U(1)_{\epsilon}$ and baryon number conservation, respectively.

The above can be generalized to $N_{f}>1$ staggered fermions. There one has a $U(1) \times U(1)$ symmetry for each flavor, leading to $U\left(N_{f}\right) \times U\left(N_{f}\right)$, which is extended to $U\left(2 N_{f}\right)$ at $\mu=0$. The same Goldstone modes listed in Table I appear also for $N_{f}>1$ (and in particular for the $N_{f}=2$ case considered in this paper), but with different multiplicities. The full symmetry-breaking pattern is summarized in Fig. 10. It can be seen that any-color QCD with quarks in the adjoint representation in the continuum exhibits the same pattern of symmetry breaking, with an additional breaking of $U(1)_{A}$ due to the axial anomaly [25].

The Goldstone spectrum consists of two meson modes, the (pseudoscalar) pion $\pi$ and the scalar meson $f_{0}$, and two diquark modes, a scalar diquark $q q$ and a pseudoscalar diquark $\epsilon q q$. In Table II, we show the employed interpolating operators for the $f_{0}$ and $\pi$ modes taken from Ref. [53]. It is important to realize that channel 1 not only contains the desired scalar meson, but also an excited pion. However, these two states can be separated during the fitting procedure as they have opposite parity. The ground state pion is exclusively contained in channel 2 . The interpolating operators from [3] are employed for the (pseudo)scalar diquark modes, shown in Table III These modes furthermore contain contributions from their corresponding antidiquarks. Nevertheless, the antidiquark modes become less important with increasing chemical potential as the propagation of

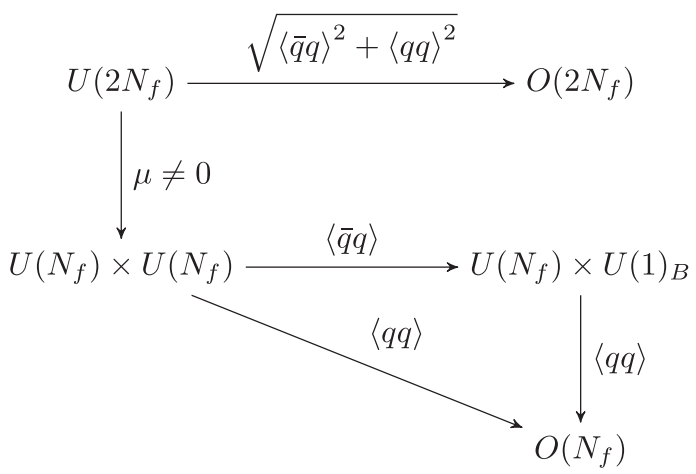

FIG. 10. Symmetry-breaking pattern of staggered action for two-color QCD at $N_{f} \geq 1$. 
TABLE II. The interpolating operators for $f_{0}$ and $\pi$ modes.

\begin{tabular}{lccc}
\hline \hline Channel & Operator & $J^{P C}$ & States \\
\hline 1 & $\bar{\chi} \chi$ & $0^{++}$ & $f_{0}$ \\
& & $0^{-+}$ & $\pi$ \\
2 & $\eta_{4} \bar{\chi} \chi$ & $0^{+-}$ & $\ldots$ \\
& & $0^{-+}$ & $\pi$ \\
\hline \hline
\end{tabular}

TABLE III. The interpolating operators for the (pseudo) scalar diquark modes.

\begin{tabular}{lcc}
\hline \hline Channel & Operator & States \\
\hline 3 & $\frac{1}{2}\left(\chi^{T} \tau_{2} \chi-\bar{\chi} \tau_{2} \bar{\chi}^{T}\right)$ & $q q / \bar{q} \bar{q}$ \\
4 & $\eta_{5} \frac{1}{2}\left(\chi^{T} \tau_{2} \chi+\bar{\chi} \tau_{2} \bar{\chi}^{T}\right)$ & $\varepsilon q q / \varepsilon \bar{q} \bar{q}$ \\
\hline \hline
\end{tabular}

particles is favored $\left(e^{a \mu}\right)$ over the propagation of antiparticles $\left(e^{-a \mu}\right)$ at nonvanishing chemical potential.

To extract the ground state masses of the particle states, we employ the zero-momentum projected correlations functions of the form,

$$
C(t)=\sum_{\vec{x}}\langle 0|O(\vec{x}, t) \bar{O}(\overrightarrow{0}, 0)| 0\rangle .
$$

Note that only the connected contributions are considered here. For the different channels shown in Tables II and III we obtain:

(i) Channel 1-scalar meson

$$
C(t)=-\sum_{\vec{x}} \eta_{5}(\vec{x}, t) \operatorname{tr}\left[G^{\dagger}[-\mu](\vec{x}, t ; 0) G[\mu](\vec{x}, t ; 0)\right]
$$

(ii) Channel 2-pion / pseudoscalar meson

$$
C(t)=-(-1)^{t} \sum_{\vec{x}} \operatorname{tr}\left[G^{\dagger}[-\mu](\vec{x}, t ; 0) G[\mu](\vec{x}, t ; 0)\right]
$$

(iii) Channel 3-scalar diquark

$$
\begin{aligned}
C(t)= & \frac{1}{2} \sum_{\vec{x}}\left\{\operatorname{tr}\left[G^{T}[\mu](\vec{x}, t ; 0) \tau_{2} G[\mu](\vec{x}, t ; 0) \tau_{2}\right]\right. \\
& \left.+\operatorname{tr}\left[G^{\dagger}[-\mu](\vec{x}, t ; 0) \tau_{2}\left(G^{\dagger}\right)^{T}[-\mu](\vec{x}, t ; 0) \tau_{2}\right]\right\}
\end{aligned}
$$

(iv) Channel 4-pseudoscalar diquark

$$
\begin{aligned}
C(t)= & \frac{1}{2} \sum_{\vec{x}} \eta_{5}(\vec{x}, t)\left\{\operatorname{tr}\left[G^{T}[\mu](\vec{x}, t ; 0) \tau_{2} G[\mu](\vec{x}, t ; 0) \tau_{2}\right]\right. \\
& \left.+\operatorname{tr}\left[G^{\dagger}[-\mu](\vec{x}, t ; 0) \tau_{2}\left(G^{\dagger}\right)^{T}[-\mu](\vec{x}, t ; 0) \tau_{2}\right]\right\} .
\end{aligned}
$$

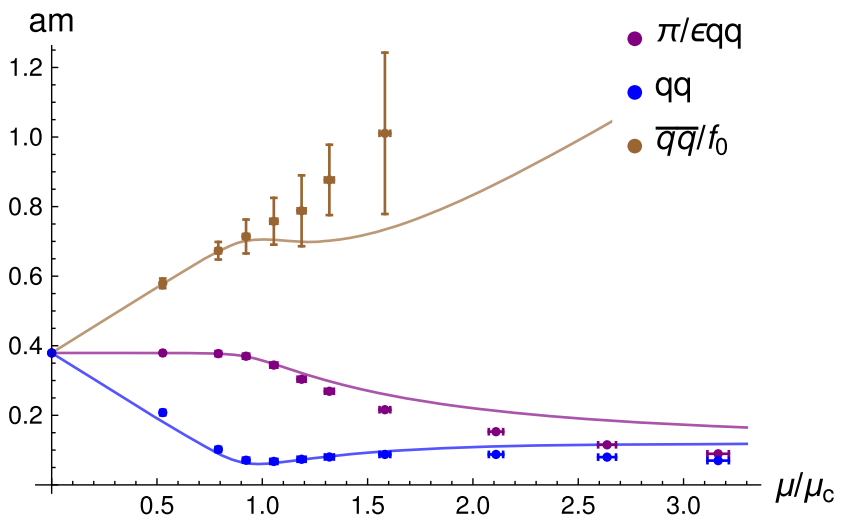

FIG. 11. The (pseudo) Goldstone spectrum on a $12^{3} \times 24$ lattice at $\beta=1.5$ with quark mass $a m=0.025$ and diquark source $\lambda=0.0025$.

We used the notation $G=\left(D^{\dagger} D+\lambda^{2}\right)^{-1} D^{\dagger}$ here, which corresponds to only the off diagonal terms in Eq. (7). The diagonal terms produce corrections of order $O\left(\lambda^{2}\right)$, which we refrain from listing here explicitly as their contributions are negligibly small for the values of $\lambda$ considered in this work. We did in fact include these corrections for the results shown in Figs. 11 and 13. The results in Fig. 12 were obtained without them.
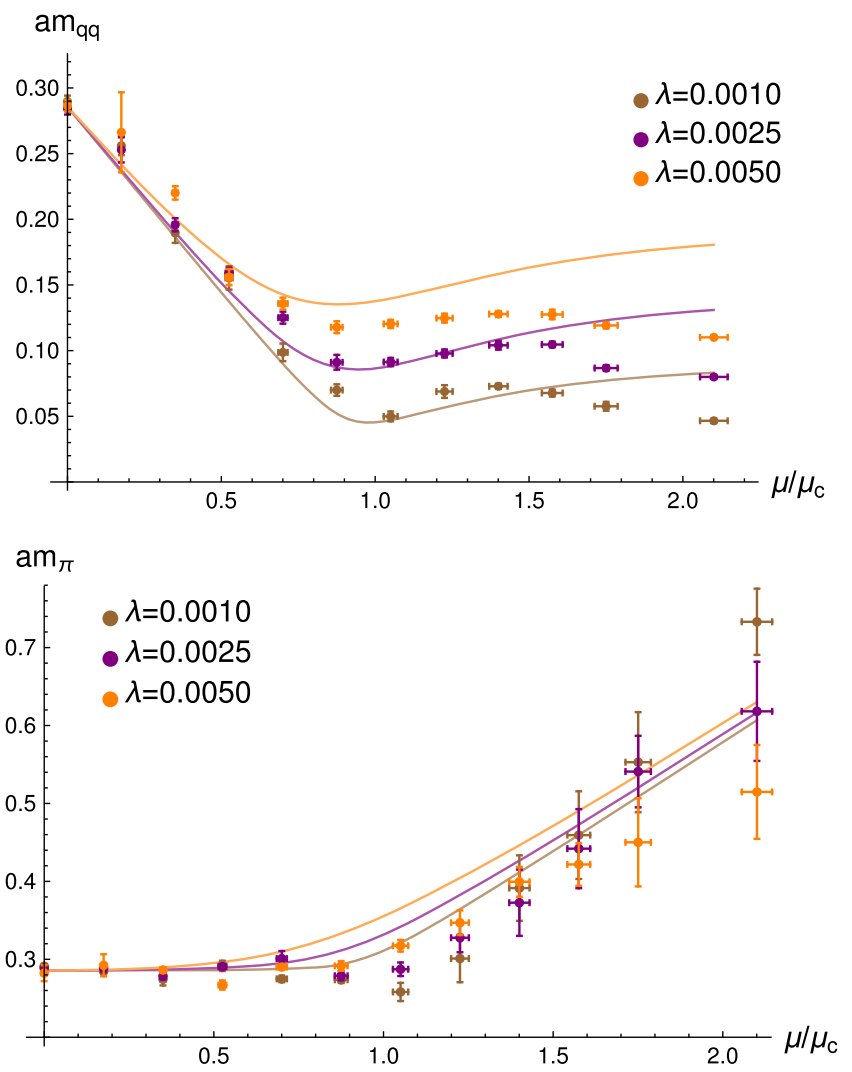

FIG. 12. The scalar diquark mass (top) and the pion mass (bottom) on a $16^{3} \times 32$ lattice at $\beta=1.7$ with quark mass am $=$ 0.01 for different diquark sources $\lambda$. 


\section{A. Goldstone spectrum in the bulk phase}

We now study the (pseudo) Goldstone spectrum on a $12^{3} \times 24$ lattice at $\beta=1.5$ with quark mass $a m=0.025$ and diquark source $\lambda=0.0025$. As the combined condensate $\sqrt{\langle\bar{q} q\rangle^{2}+\langle q q\rangle^{2}}$ rotates from a chiral to a diquark condensate with increasing chemical potential, we assume that the Goldstone modes corresponding to a given $U(2)$ generator mix in a similar way, i.e., rotate into each other with the same rotation angle $\alpha(\mu)$, as described by Eq. (9). Hence, we introduce the two combined modes,

$\bar{q} \bar{q} / f_{0}: \frac{1}{2}\left(\chi^{T} \tau_{2} \chi+\bar{\chi} \tau_{2} \bar{\chi}^{T}\right) \cos \alpha+\bar{\chi} \chi \sin \alpha$

$\pi / \epsilon q q: \bar{\chi} \epsilon \chi \cos \alpha+\frac{1}{2}\left(\chi^{T} \tau_{2} \epsilon \chi+\bar{\chi} \tau_{2} \epsilon \bar{\chi}^{T}\right) \sin \alpha$.

Note that this simple mode mixing holds only to leading order $\chi \mathrm{PT}$. Note also that in addition to the terms of order $O\left(\lambda^{2}\right)$ discussed above, the correlators of the combined modes $\bar{q} \bar{q} / f_{0}$ and $\pi / \epsilon q q$ contain additional terms of order $O(\lambda)$ from the mixed parts (i.e., the terms $\sim \sin \alpha \cos \alpha$ ), as diagonal elements of the full propagator in Nambu-Gorkov space (7) contribute to these. We again refrain from listing these terms here explicitly but included them in our simulations.

To obtain the masses of the (pseudo) Goldstone modes, we measure the zero-momentum projected connected correlation functions and extract their masses from fits to $\cosh (\cdot)$, which combines exponential decays forwards and backwards in Euclidean time. ${ }^{4}$ For $\pi / \epsilon q q$ a factor $(-1)^{t}$ is inserted to account for negative parity. In the case of the $\bar{q} \bar{q} / f_{0}$ mode we have to use the fitting function,

$$
\begin{aligned}
C(t)= & A \cosh \left(m_{q q}\left(t-\frac{N_{t}}{2}\right)\right) \\
& +B \cosh \left(m_{\bar{q} \bar{q} / f_{0}}\left(t-\frac{N_{t}}{2}\right)\right)
\end{aligned}
$$

as the correlator contains a contribution from the scalar diquark mode $q q$ in addition to the mixing of the scalar antidiquark $\bar{q} \bar{q}$ with the scalar meson $f_{0}$.

At $\mu \neq 0$ the combined mode $\bar{q} \bar{q} / f_{0}$ is a massive pseudo Goldstone mode for all values of $\mu$. Including a nonvanishing quark mass $m \neq 0$, also the combined mode $\pi / \epsilon q q$ becomes a pseudo Goldstone mode as the $U(1)_{A}$ symmetry gets broken. The only true Goldstone mode is given by the scalar diquark mode $q q$ in the limit $\lambda \rightarrow 0$ and for $\mu>\mu_{\mathrm{c}}$. Generally for $\mu<\mu_{\mathrm{c}}$ and $\lambda \rightarrow 0$, the pion mass $m_{\pi}$ stays constant as the pion does not carry a net Baryon number, whereas the scalar diquark mass $m_{q q}$ decreases like $m_{\pi}-2 \mu$ and the scalar antidiquark mass $m_{\bar{q}} \bar{q}$ increases like $m_{\pi}+2 \mu$.

We compare our obtained masses of the (pseudo) Goldstone modes to the corresponding $\chi \mathrm{PT}$ predictions for two color QCD with staggered quarks [3], using $\mu_{c}$ from

\footnotetext{
${ }^{4}$ We can use $\cosh (\cdot)$ also for the diquark channels (3 and 4), as time-reversal asymmetric contributions to the correlators cancel due to mixing with antidiquarks.
}

the fit of the condensates in Sec. III (see Fig. 11) and the lattice parameters $a \lambda$ and $a m$ as input. The large error of the combined mode $\bar{q} \bar{q} / f_{0}$ mainly comes from the systematic error as the double-cosh fit is more sensitive to the fitting interval than the single-cosh fits of the other modes.

We find a general agreement of the scalar diquark mode $q q$ and the $\pi / \epsilon q q$ mode to their predictions. Deviations become notable at large chemical potential, where also the quark number density in Fig. 2 deviates largely. The large discrepancy of the $\bar{q} \bar{q} / f_{0}$ mode for $\mu \geq \mu_{\mathrm{c}}$ might be due to omitting disconnected contributions, as at large chemical potential the scalar meson mode $f_{0}$ dominates this combined mode. Note that the scalar diquark mode $q q$ has disconnected contributions of order $O\left(\lambda^{2}\right)$, which we expect to have a small effect. Hence, we obtain similar results as in the previous study [3] for $N_{\mathrm{f}}=4$. However, in Ref. [3] disconnected contributions have not been omitted, and thus their obtained $\bar{q} \bar{q} / f_{0}$ mode coincides better with the $\chi \mathrm{PT}$ prediction. In conclusion, we find that within the bulk phase fundamental staggered quarks resemble the chiral symmetry breaking pattern of adjoint QCD or $G_{2}-\mathrm{QCD}$ in the continuum as most notably seen in the behavior of the pion branch above the onset at $a \mu_{\mathrm{c}}$.

\section{B. Goldstone spectrum outside bulk phase}

Continuum two-color QCD with quarks in the fundamental representation obeys the pattern of symmetry breaking $S U\left(2 N_{\mathrm{f}}\right) \rightarrow S p\left(2 N_{\mathrm{f}}\right)$. Applying $\chi \mathrm{PT}$, it was found that the mass of the pion mode increases for $\mu>\mu_{\mathrm{c}}$ [25] in this case, due to a swapping of the $P_{S}$ and $P_{A}$ branches when compared to the staggered action [3]. We now wish to test whether the pattern of symmetry breaking on the lattice will change to the continuum pattern in the limit $a \rightarrow 0$. Thus, we calculate the spectrum again at a larger inverse gauge coupling and using an improved gauge action.

With the new parameters $\left(\beta=1.7, N_{s}=16\right.$ and $N_{t}=32$ ), where monopoles are strongly suppressed, we find a quite different behavior of the (pseudo) Goldstone modes than in Sec. VI A. We observe that the combined modes $\bar{q} \bar{q} / f_{0}$ and $\pi / \epsilon q q$ do not give any meaningful results. Instead, we therefore study the scalar diquark mode $q q$ and the pion mode $\pi$ individually, and additionally we extract the mass of the scalar antidiquark $\bar{q} \bar{q}$ from the operator $\chi^{T} \tau_{2} \chi-\bar{\chi} \tau_{2} \bar{\chi}^{T}$ for $\mu<\mu_{\mathrm{c}}$. In the correlation function of the pion mode we find an additional contribution from an opposite parity state, which we filter out by applying a double cosh-fit of the form,

$$
\begin{aligned}
C(t)= & A \cosh \left(m_{\pi}\left(t-\frac{N_{t}}{2}\right)\right) \\
& +(-1)^{t} B \cosh \left(m_{\pi^{\star}}\left(t-\frac{N_{t}}{2}\right)\right) .
\end{aligned}
$$






FIG. 13. The (pseudo) Goldstone spectrum on a $16^{3} \times 32$ lattice at $\beta=1.7$ with quark mass $a m=0.01$ and diquark source $\lambda=0.001$.

We first study the $\lambda$-dependence pion mass and the scalar diquark mass (see Fig. 12). Here we neglected $O\left(\lambda^{2}\right)$ contributions from the diagonal terms in Eq. (7) in the calculation of the correlation functions. We compare the results to $\chi \mathrm{PT}$ predictions for from continuum twocolor QCD with quarks in the fundamental representation, for which we use the lattice parameters $\lambda$ and $m$, and also $\mu_{\mathrm{c}}$ as obtained from measuring the pion mass at $\mu=0$, as input. For the smallest diquark source $\lambda=0.001$ we find excellent agreement with $\chi \mathrm{PT}$ up to $\mu \sim 1.7 \mu_{c}$ where leading order $\chi \mathrm{PT}$ is not reliable anymore. We conclude that the diagonal terms are negligible for this choice of diquark source.

At $\beta=1.7$ the pion mass stays constant for $\mu \leq \mu_{\mathrm{c}}$, but it starts to increase for $\mu>\mu_{\mathrm{c}}$. This differs profoundly from the behavior at $\beta=1.5$ (cf. Fig 11). Again, the large error of the pion mode $\pi$ for $\mu>\mu_{\mathrm{c}}$ mainly comes from the sensitivity of the double-cosh fit to the fitting interval. We interpret the increasing pion mass for $\mu>\mu_{\mathrm{c}}$ as strong evidence that the pattern of symmetry breaking changed to its continuum counterpart outside of the bulk phase. Figure 13 combines the results for all the considered meson channels, for $\lambda=0.001$ where the agreement with $\chi \mathrm{PT}$ is nearly perfect. In this figure the $O\left(\lambda^{2}\right)$ diagonal terms were in fact included, but their contribution is of similar magnitude as the statistical error.

\section{UNFOLDED LEVEL SPACINGS}

The low energy features of QCD are dominated by its global symmetries. In this limit, QCD is well described with only the two lightest quarks. Low-energy QCD can be approximated with a random matrix theory very well, where all interactions among the degrees of freedoms of the theory are equally likely and completely determined by the global symmetries. In a random matrix theory (RMT), the matrix elements of the Dirac operator are replaced with uncorrelated random numbers, in a way such

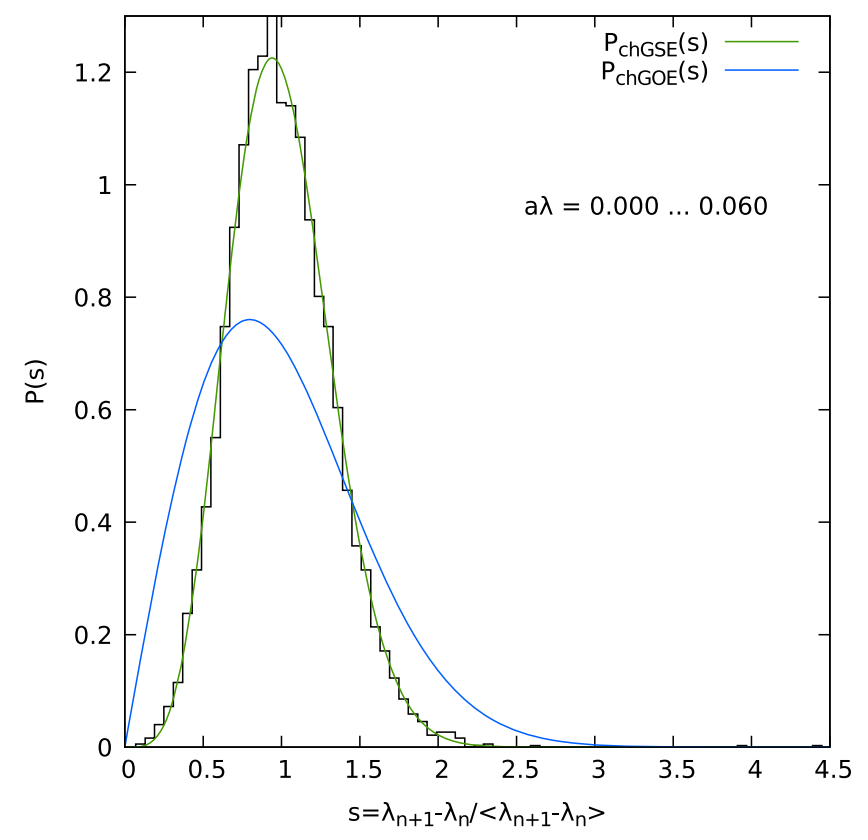

FIG. 14. The unfolded level spacing distribution of the staggered Dirac operator spectrum inside the bulk phase (unimproved action, $\left.12^{3} \times 24, \beta=1.5\right)$. The level spacings are entirely distributed according to the RMT prediction for the chiral symplectic ensemble.

that the global symmetries of the operator prevail [54]. Any observable is then an average over random matrix elements and depends only on universal features of the theory, and not on microscopic interactions.

RMT has found wide application for studying the eigenvalue statistics in nuclear resonances [55]. The distribution of level spacings is cleared off of any microscopic interactions by rescaling the spacings such that their average is unity and removing the fluctuating part of the cumulative spectral function. This procedure is called unfolding. We wish to demonstrate here that the change of the Goldstone spectrum of two color QCD with staggered fermions when leaving the bulk phase is accompanied by a change of the unfolded level spacing distribution (ULSD) of the Dirac operator.

For different random matrix ensembles different forms of ULSD have been predicted [56,57]. For the Gaussian orthogonal ensemble (GOE), where the matrix elements are real and the Dyson index is $\beta_{D}=1$, the ULSD is

$$
P_{\beta_{D}=1}(s)=\frac{\pi}{2} s e^{-\frac{\pi}{4} s^{2}} .
$$

For the Gaussian symplectic ensemble (GSE) the matrix elements are quaternion real and the Dyson index is $\beta_{D}=4$. The ULSD of the GSE is given by

$$
P_{\beta_{D}=4}(s)=\left(\frac{8}{3}\right)^{6} \frac{1}{\pi^{3}} s^{4} e^{-\left(\frac{8}{3}\right)^{2} \frac{1}{\pi^{3}} s^{2}} .
$$




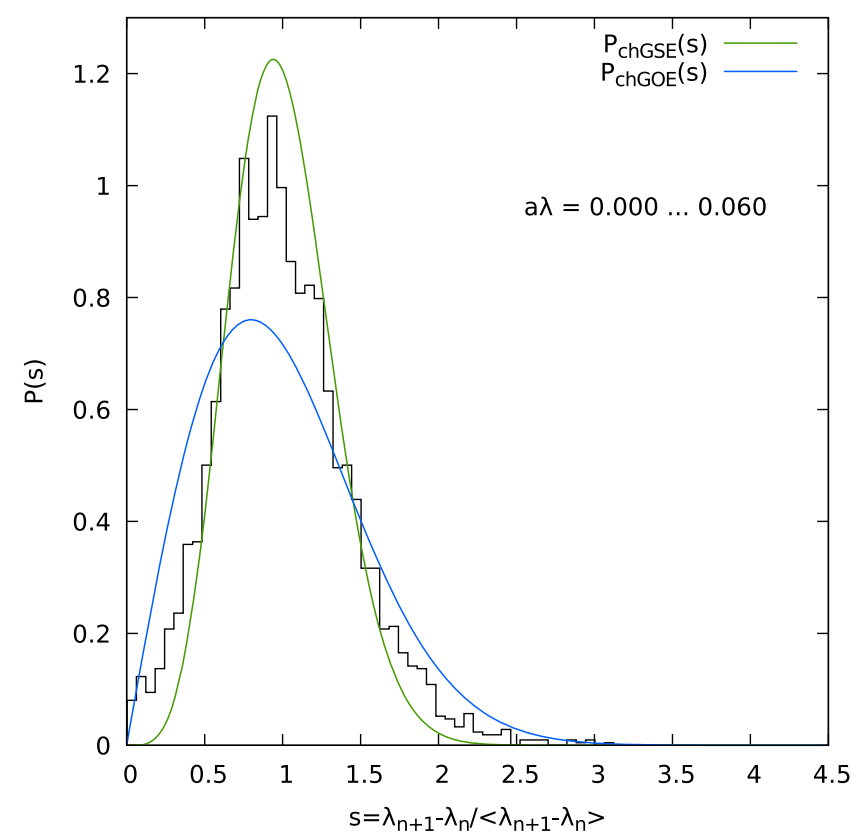

FIG. 15. The unfolded level spacing distribution of the staggered Dirac operator outside the bulk phase (Symanzik action, $\left.16^{3} \times 32, \beta=1.7\right)$. Neither GSE nor GOE describe the distribution entirely.

Using the implicitly restarted Arnoldi algorithm, we have measured the ULSD both with the parameters from Ref. [3] $\left(12^{3} \times 24, a m=0.025, \beta=1.5\right.$, Wilson action $)$ within the bulk phase, as well as with our improved parameters $\left(16^{3} \times 32\right.$, am $=0.01 \beta=1.7$, Symanzik action $)$ on the weak-coupling side of the bulk crossover transition.

The ULSD within the bulk phase are shown in Fig. 14. We find that the entire spectral range is well described by Eq. (27); i.e., the distributions resembles the symplectic ensemble very well. Inside the bulk phase, the ULSD does not seem to contain any component distributed according to the orthogonal ensemble.

In the continuum limit the spectrum is expected to resemble the Gaussian orthogonal ensemble. With our improved parameters, we find however that neither Eq. (27) nor Eq. (26), fully describe the ULSD. In fact, our numerical data (seen in Fig. 15) indicate some sort of intermediate state. When separating the low-lying $(a \lambda=0.000-0.036)$ from the high-lying eigenmodes $(a \lambda=0.040-0.160)$ it becomes clear that a large part of the low eigenmodes are now distributed according to the GOE, while the higher eigenmodes remain distributed according to the GSE (see Fig. 16). Since the low eigenmodes govern the Goldstone modes, it is clear that our spectroscopic results should reflect the chiralsymmetry breaking pattern of the GOE. We suspect that in the continuum limit the symplectic part will vanish entirely.
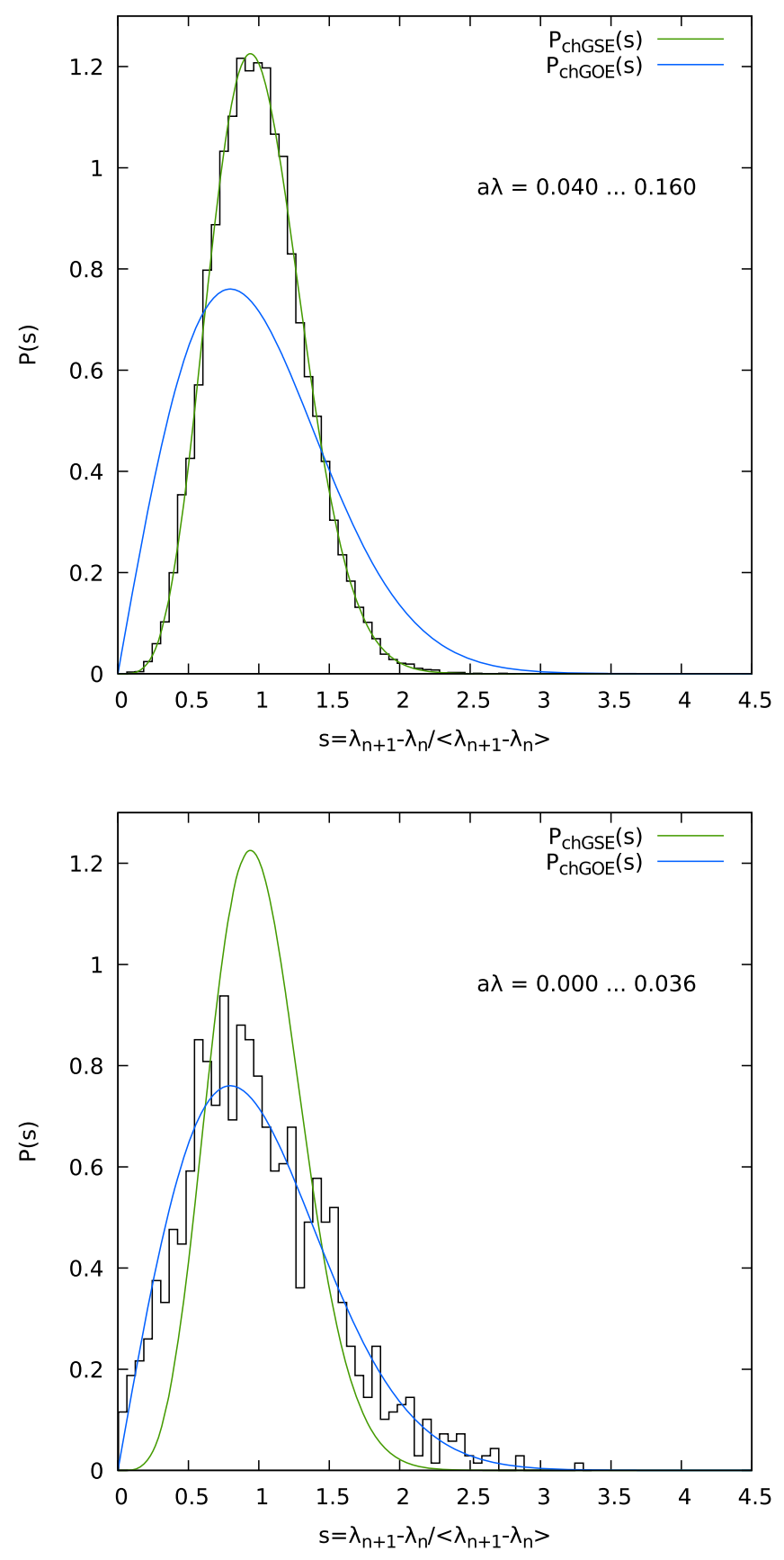

FIG. 16. The unfolded level spacing distribution of the staggered Dirac operator outside the bulk phase (Symanzik action, $\left.16^{3} \times 32, \beta=1.7\right)$. Low and high modes shown separately. Highlying modes (top) are described by GSE while low-lying modes (bottom) are described by GOE.

\section{CONCLUSION AND OUTLOOK}

In this work we investigated the influence of bulk effects in $\mathrm{QC}_{2} \mathrm{D}$ with staggered fermions on the Goldstone spectrum and the unfolded level spacing distribution of the Dirac operator at finite density. We compared the 
Goldstone spectrum to predictions from leading order chiral perturbation theory for two sets of lattice parameters:

(i) A $12^{3} \times 24$ lattice with $\beta=1.5$ and bare mass $a m=$ 0.025 using a standard Wilson gauge action. The density of $Z_{2}$ monopoles at $\mu=0$ is $\langle z\rangle \sim 0.88$ in this case.

(ii) A $16^{3} \times 32$ lattice with $\beta=1.7$ and bare mass $a m=$ 0.01 using a treelevel improved Symanzik gauge action. The density of $Z_{2}$ monopoles at $\mu=0$ is $\langle z\rangle \sim 0.27$ in this case.

Our main result is that the Goldstone spectrum switches from that of any-color QCD with adjoint fermions in the bulk phase to that of two color QCD with fundamental quarks on the physical weak-coupling side of the bulk crossover, as most notably visible in a change of the pion branch. We show that this change is reflected in the unfolded level spacing distribution, which appears to obtain a larger and larger contribution from the Gaussian orthogonal random-matrix ensemble, starting with the low-lying eigenmodes, as one moves from strong to weak compling, while deeply in the bulk phase the distribution is completely dominated by the Gaussian symplectic ensemble. We conclude that a continuum limit leading to two color QCD with the correct chiral symmetry-breaking pattern is possible with rooted staggered quarks.

We also observed that the standard connected susceptibility subtraction to obtain a renormalized chiral condensate cannot be used at finite $\mu$, since the connected susceptibility contains a singular contribution at the diquark condensation transition and that a renormalization using a heavy quark condensate is also rendered unfeasible. Developing a proper $\mu$-dependent renormalization scheme might be possible using gradient flow techniques, but is left for future work.

\section{ACKNOWLEDGMENTS}

This work was supported by the Helmholtz International Center for FAIR within the LOEWE initiative of the State of Hesse.
[1] A. Maas, L. von Smekal, B. Wellegehausen, and A. Wipf, The phase diagram of a gauge theory with fermionic baryons, Phys. Rev. D 86, 111901 (2012).

[2] B. H. Wellegehausen, A. Maas, A. Wipf, and L. von Smekal, Hadron masses and baryonic scales in $G_{2}$-QCD at finite density, Phys. Rev. D 89, 056007 (2014).

[3] J. B. Kogut, D. Toublan, and D. K. Sinclair, The PseudoGoldstone spectrum of two color QCD at finite density, Phys. Rev. D 68, 054507 (2003).

[4] S. Hands, J. B. Kogut, M.-P. Lombardo, and S. E. Morrison, Symmetries and spectrum of SU(2) lattice gauge theory at finite chemical potential, Nucl. Phys. B558, 327 (1999).

[5] S. Hands, I. Montvay, S. Morrison, M. Oevers, L. Scorzato, and J. Skullerud, Numerical study of dense adjoint matter in two color QCD, Eur. Phys. J. C 17, 285 (2000).

[6] J. B. Kogut, D. Toublan, and D. K. Sinclair, Diquark condensation at nonzero chemical potential and temperature, Phys. Lett. B 514, 77 (2001).

[7] J. B. Kogut, D. K. Sinclair, S. J. Hands, and S. E. Morrison, Two color QCD at nonzero quark number density, Phys. Rev. D 64, 094505 (2001).

[8] S. Chandrasekharan and F.-J. Jiang, Phase-diagram of two-color lattice QCD in the chiral limit, Phys. Rev. D 74, 014506 (2006).

[9] V. V. Braguta, E. M. Ilgenfritz, A. Yu. Kotov, A. V. Molochkov, and A. A. Nikolaev, Study of the phase diagram of dense two-color QCD within lattice simulation, Phys. Rev. D 94, 114510 (2016).

[10] L. Holicki, J. Wilhelm, D. Smith, B. Wellegehausen, and L. von Smekal, Two-colour QCD at finite density with two flavours of staggered quarks, Proc. Sci., LATTICE2016 (2017) 052 .
[11] V. G. Bornyakov, V. V. Braguta, E. M. Ilgenfritz, A. Yu. Kotov, A. V. Molochkov, and A. A. Nikolaev, Observation of deconfinement in a cold dense quark medium, J. High Energy Phys. 03 (2018) 161.

[12] N. Yu. Astrakhantsev, V. G. Bornyakov, V. V. Braguta, E. M. Ilgenfritz, A. Yu. Kotov, A. A. Nikolaev, and A. Rothkopf, Lattice study of static quark-antiquark interactions in dense quark matter, J. High Energy Phys. 05 (2019) 171.

[13] S. Hands, S. Kim, and J.-I. Skullerud, Deconfinement in dense 2-color QCD, Eur. Phys. J. C 48, 193 (2006).

[14] S. Hands, S. Kim, and J.-I. Skullerud, A quarkyonic phase in dense two color matter?, Phys. Rev. D 81, 091502 (2010).

[15] S. Hands, P. Kenny, S. Kim, and J.-I. Skullerud, Lattice study of dense matter with two colors and four flavors, Eur. Phys. J. A 47, 60 (2011).

[16] S. Cotter, P. Giudice, S. Hands, and J.-I. Skullerud, Towards the phase diagram of dense two-color matter, Phys. Rev. D 87, 034507 (2013).

[17] T. Boz, P. Giudice, S. Hands, J.-I. Skullerud, and A. G. Williams, Two-color QCD at high density, AIP Conf. Proc. 1701, 060019 (2016).

[18] E. Itou, K. Iida, and T.-G. Lee, Topology of two-color QCD at low temperature and high density, Proc. Sci., LATTICE2018 (2018) 168.

[19] D. T. Son and M. A. Stephanov, QCD at Finite Isospin Density, Phys. Rev. Lett. 86, 592 (2001).

[20] W. Detmold, K. Orginos, and Z. Shi, Lattice QCD at nonzero isospin chemical potential, Phys. Rev. D 86, 054507 (2012).

[21] K. Kamikado, N. Strodthoff, L. von Smekal, and J. Wambach, Fluctuations in the quark-meson model for 
QCD with isospin chemical potential, Phys. Lett. B 718, 1044 (2013).

[22] I. Boettcher, T. K. Herbst, J. M. Pawlowski, N. Strodthoff, L. von Smekal, and C. Wetterich, Sarma phase in relativistic and non-relativistic systems, Phys. Lett. B 742, 86 (2015).

[23] B. B. Brandt, G. Endrodi, and S. Schmalzbauer, QCD phase diagram for nonzero isospin-asymmetry, Phys. Rev. D 97, 054514 (2018).

[24] J. B. Kogut, M. A. Stephanov, and D. Toublan, On two color QCD with baryon chemical potential, Phys. Lett. B 464, 183 (1999).

[25] J. Kogut, M. A. Stephanov, D. Toublan, J. Verbaarschot, and A. Zhitnitsky, QCD — like theories at finite baryon density, Nucl. Phys. B582, 477 (2000).

[26] K. Splittorff, D. Toublan, and J. Verbaarschot, Diquark condensate in QCD with two colors at next-to-leading order, Nucl. Phys. B620, 290 (2002).

[27] T. Brauner, On the chiral perturbation theory for two-flavor two-color QCD at finite chemical potential, Mod. Phys. Lett. A 21, 559 (2006).

[28] T. Kanazawa, T. Wettig, and N. Yamamoto, Chiral Lagrangian and spectral sum rules for dense two-color QCD, J. High Energy Phys. 08 (2009) 003.

[29] T. Kanazawa, T. Wettig, and N. Yamamoto, Chiral random matrix theory for two-color QCD at high density, Phys. Rev. D 81, 081701 (2010).

[30] T. Kanazawa, T. Wettig, and N. Yamamoto, Singular values of the Dirac operator in dense QCD-like theories, J. High Energy Phys. 12 (2011) 007.

[31] G.-f. Sun, L. He, and P. Zhuang, BEC-BCS crossover in the Nambu-Jona-Lasinio model of QCD, Phys. Rev. D 75, 096004 (2007).

[32] L. He, Nambu-Jona-Lasinio model description of weakly interacting Bose condensate and BEC-BCS crossover in dense QCD-like theories, Phys. Rev. D 82, 096003 (2010).

[33] N. Strodthoff, B.-J. Schaefer, and L. von Smekal, Quarkmeson-diquark model for two-color QCD, Phys. Rev. D 85, 074007 (2012).

[34] N. Strodthoff and L. von Smekal, Polyakov-Quark-MesonDiquark Model for two-color QCD, Phys. Lett. B 731, 350 (2014).

[35] M. Golterman, Y. Shamir, and B. Svetitsky, Breakdown of staggered fermions at nonzero chemical potential, Phys. Rev. D 74, 071501 (2006).

[36] M.F. L. Golterman and J. Smit, Selfenergy and flavor interpretation of staggered fermions, Nucl. Phys. B245, 61 (1984).

[37] B. H. Wellegehausen and L. von Smekal, Lattice simulations of $G_{2}$-QCD at finite density, Proc. Sci., LATTICE2014 (2015) 177.

[38] D. Scheffler, Two-color lattice qcd with staggered quarks, Ph.D thesis, TU Darmstadt, 2015.

[39] G. Bhanot and M. Creutz, Variant actions and phase structure in lattice gauge theory, Phys. Rev. D 24, 3212 (1981).
[40] I. G. Halliday and A. Schwimmer, The phase structure of $\mathrm{SU}(\mathrm{N}) / \mathrm{Z}(\mathrm{N})$ lattice gauge theories, Phys. Lett. 101B, 327 (1981).

[41] I. G. Halliday and A. Schwimmer, Z(2) monopoles in lattice gauge theories, Phys. Lett. 102B, 337 (1981).

[42] G. Mack and V. B. Petkova, Z2 monopoles in the standard su(2) lattice gauge theory model, Z. Phys. C 12, 177 (1982).

[43] A. Barresi, G. Burgio, and M. Mueller-Preussker, SO(3) versus SU(2) Yang-Mills theory on the lattice: An Investigation at nonzero temperature, Proceedings Confinement 2003, 82 (2003).

[44] U. M. Heller, SU(3) lattice gauge theory in the fundamental adjoint plane and scaling along the Wilson axis, Phys. Lett. B 362, 123 (1995).

[45] S. Aoki et al. (JLQCD collaboration), Bulk first-order phase transition in three-flavor lattice QCD with $\mathrm{O}(\mathrm{a})$-improved Wilson fermion action at zero temperature, Phys. Rev. D 72, 054510 (2005).

[46] F. R. Brown, H. Chen, N. H. Christ, Z. Dong, R. D. Mawhinney, W. Schaffer, and A. Vaccarino, Lattice QCD with eight light quark flavors, Phys. Rev. D 46, 5655 (1992).

[47] P. Weisz and R. Wohlert, Continuum limit improved lattice action for pure yang-mills theory. 2., Nucl. Phys. B236, 397 (1984).

[48] W. Unger, The chiral phase transition of QCD with $2+1$ flavors, Ph.D thesis, Univ. Bielefeld, 2010.

[49] P. Scior and L. von Smekal, Baryonic matter onset in twocolor qcd with heavy quarks, Phys. Rev. D 92, 094504 (2015).

[50] P. Scior and L. von Smekal, Effective Polyakov loop models for QCD-like theories at finite chemical potential, Proc. Sci., LATTICE2015 (2016) 205 [arXiv:1602.04614].

[51] B. H. Wellegehausen and L. von Smekal, What we can learn from two-dimensional QCD-like theories at finite density, Proc. Sci., LATTICE2016 (2016) 078 [arXiv:1702.00238].

[52] V. G. Bornyakov, V. V. Braguta, E. M. Ilgenfritz, A. Yu. Kotov, I. E. Kudrov, A. V. Molochkov et al., Confinementdeconfinement transition in dense SU(2) QCD, EPJ Web Conf. 175, 07009 (2018).

[53] R. Altmeyer, K. Born, M. Gckeler, E. Laermann, and G. Schierholz, The hadron spectrum in qcd with dynamical staggered fermions, Nucl. Phys. B389, 445 (1993).

[54] J. J. M. Verbaarschot and T. Wettig, Random matrix theory and chiral symmetry in QCD, Annu. Rev. Nucl. Part. Sci. 50, 343 (2000).

[55] M. L. Mehta, Random Matrices, 3rd ed. (Elsevier Academic Press, New York, 2004).

[56] F. Bruckmann, S. Keppeler, M. Panero, and T. Wettig, Polyakov loops and spectral properties of the staggered Dirac operator, Phys. Rev. D 78, 034503 (2008).

[57] P. C. Huu-Tai, N. A. Smirnova, and P. Van Isacker, Generalised Wigner surmise for, random matrices $(2 \times 2)$, J. Phys. A: Math. Gen. (to be published). 